\title{
HANDBALL-SPECIFIC SKILL ACQUISITION BY USE OF DIFFERENT INSTRUCTION METHODS
}

original paper

( ) University School of Physical Education in Wroclaw

DOI: https://doi.org/10.5114/hm.2021.100323

\section{FROWIN FASOLD, LUCA HOUSEMAN, BENJAMIN NOËL, STEFANIE KLATT}

German Sport University Cologne, Cologne, Germany

\section{ABSTRACT}

Purpose. Instructions are one of the fundamental coaching methods for developing skills in sport. Recent research has shown that activating implicit learning processes through metaphorical or analogical instructions is as effective as the activation of explicit learning processes by instructing step-by-step movement rules. Nevertheless, these effects have not been investigated in youth handball yet, and past research has shown a lack of the systematic use of analogy instructions in general. For the first time, the current study investigated the effectiveness of step-by-step instructions and analogy instructions in young athletes' learning of basic skills in team handball.

Methods. A short-term intervention with a sample of 36 participants (9-10 years) was conducted. One group of the athletes were instructed with step-by-step movement rules, while the other group were instructed with analogies, in 3 different handball-specific tasks (stand throw, body feint, defence position). The athletes' performances were rated by 15 handball coaches.

Results. The conducted intervention resulted in small and task-dependent performance improvements under both instruction conditions. In the throwing task, no performance improvement was observed overall, whereas the participants improved in both instruction conditions in the feint task. As for the learning of the defence position, the participants in the analogy condition improved more than the others.

Conclusions. The current results are in line with recent investigations of different sports tasks and should motivate coaches and teachers in team sports to try to be ingenious and creative in developing functional analogies, metaphors, or pictorial instructions for skill acquisition.

Key words: coaching, teaching, implicit learning, explicit learning

\section{Introduction}

Sports games like handball are highly dynamic and usually require high levels of complex skills of athletes $[1,2]$. To comply with these multifarious requirements, teachers and coaches need optimal strategies for developing highly efficient teaching-learning concepts to prepare their athletes as best as possible. As Raab et al. [3] illustrated, there are various approaches to skill acquisition in team sports which are often assigned to either implicit or explicit learning. Tzetzis and Lola [4] argue that some research highlights the effectivity of explicit step-by-step learning, while different studies confirm the effectivity of implicit or analogical learning processes. Nevertheless, a direct com- parison of these learning strategies in handball is still lacking. Thus, the aim of the current study was to evaluate the effects of explicit learning rules compared with visual, implicit instructions on specific skill acquisition in handball.

The quality and quantity of training (sessions) are probably most important for the development of performance in athletes [5]. In the context of these practising processes, instructions, next to environmental parameters or demonstrations, play a major role in optimal skill learning [5]. These instructions influence/direct the learner's attentional focus [6] and are therefore able to initiate either implicit or explicit learning processes. Whereas research on a basic psychological level has demonstrated that these processes

Correspondence address: Frowin Fasold, Institute of Exercise Training and Sport Informatics, German Sport University Cologne, Am Sportpark Müngersdorf 6, 50933 Cologne, Germany, e-mail: f.fasold@dshs-koeln.de

Received: October 2, 2019

Accepted for publication: May 14, 2020

Citation: Fasold F, Houseman L, Noël B, Klatt S. Handball-specific skill acquisition by use of different instruction methods. Hum Mov. 2021;22(3):45-53; doi: https://doi.org/10.5114/hm.2021.100323. 


\section{HUMAN MOVEMENT}

F. Fasold, L. Houseman, B. Noël, S. Klatt, Instructions in handball

should not be considered independent of each other because they are directly connected [7], for coaching practice, a separation seems to be useful. If these processes are considered separately, implicit learning could be characterized by a limited requirement on working memory as well as declarative knowledge and further by stable performances under stressful conditions and secondary tasks [8]. Explicit learning could be characterized by higher requirements on working memory, an increased creation of declarative knowledge, and poorer performance under stress and secondary-task conditions [8]. According to the systematic review by Kal et al. [9], analysing 39 direct comparisons of explicit and implicit motor learning processes, there is just a small positive effect on the automaticity of motor abilities when the learning process is implicit. Nevertheless, the authors emphasized that depending on several variables (task, experiences, preference of motor control, working memory capacity), sometimes explicit, sometimes implicit teaching approaches are more promising. Moreover, analogy learning seems to be the most promising strategy for implicit learning in sports practice [9]. Interestingly, analogies are used rather rarely and certainly not systematically in coaching practice. This is probably the case because, firstly, there is currently no list of evaluated analogies amongst many different options for the instructions of body movements [10] and, secondly, the application of effective and functional analogies requires much ingenuity [9].

Whereas extensive research evaluating the effects of analogies in motor learning exists already [9], there is just a small number of different sports, namely, table tennis, golf, and basketball, in which applications of analogies have been evaluated so far [11-13]. That is, research studies investigating motor learning in the area of handball are still lacking.

On the basis of the most current comparison of implicit and explicit learning methods across several tasks [9], we assumed that implicit instructions (i.e., analogies) would have the same effect on the performance in motor learning of handball-specific skills as explicit step-by-step instructions. In more detail, we tried to explore analogies for basic skill development in handball since Masters [10] stated that no list of functional analogies in sport exists until now. Considering the complex requirements of handball [1, 2], we focused on 3 different basic skills: the stand throw, the body feint, and the basic position in defence. The instructions focused on $2 \mathrm{key}$ performance parameters of each of these movements (stand throw: shoulder position, elbow height; body feint: attacking the space on one side, fast movement to the other side; defence position: inclined position, raised arms with bent elbows).

\section{Material and methods}

\section{Design}

We conducted a study with a 3 (task: throw vs. feint $v$ s. defence position $) \times 3$ (learning phase: pre- to post- $v$ s. pre- to retention- $v$ s. post- to retention-test $) \times 2$ (instruction: step-by-step vs. analogies) design. The participating athletes were videotaped, and their performances were post-hoc rated by handball experts who were naive regarding the purpose of the study. Thus, we investigated the effects of the within-subject factors of task and learning phase, and the between-subject factor of instruction on the dependent variable of performance improvements (rated by independent experts).

\section{Participants}

\section{Participating athletes}

A total of 36 athletes (12 female, 24 male, $M_{\text {age }}=$ 9.30 years, $S D=0.71$ years) took part in the study. All of them were experienced in playing handball (5 months to 3 years). The male and female athletes were separated and were subsequently randomly assigned to the groups of step-by-step and analogy instructions, by drawing a card. The athletes' head coaches reviewed this random group assignment subjectively, by estimating the performance levels of both groups, and verified that the skill level in both groups was approximately equal. Further, no differences in the physical characteristics between the groups were obvious (step-by-step group: $M_{\text {height }}=143.17 \mathrm{~cm}, S D=8.59$, $M_{\text {weight }}=33.33 \mathrm{~kg}, S D=5.56$; analogy group: $M_{\text {height }}=$ $142.77 \mathrm{~cm}, S D=8.88, M_{\text {weight }}=34.69 \mathrm{~kg}, S D=4.13$ ). All athletes were naive regarding the purpose of the study.

\section{Participating raters}

Overall, 15 independent male handball experts ( $M_{\text {age }}=24.80$ years, $S D=7.73$ years) were responsible for the post-hoc performance ratings of the task performances. They were all experienced in coaching handball on kid and junior levels (9 of them held a C-licence and 2 held a B-licence of the German Handball Federation). 


\section{Procedure}

\section{Tests}

All athletes were more or less familiar with the 3 movement tasks. Thus, these tasks were not completely new for them. However, one has to keep in mind that usually, at this age level, only the stand throw skill is practised more intensely. The procedure and the instructions were identical in each testing/measurement session (pre-, post-, retention-test). The athletes were instructed on the tasks as follows: for the stand throw: 'Perform a stand throw and shoot a goal,' for the body feint: 'Perform a body feint in front of an Air-Body and shoot a goal,' and for the defence position: 'Defend the attacker and get in possession of the ball'. For the stand throw task, the athletes started at the 9-m line with the ball in hand and threw at the goal guarded by a goalkeeper. For the body feint task, an Air-Body was placed on the 9-m line and, after a run-up involving a double pass with the coach, the athletes performed a body feint and threw at the goal. For the defence position task, athletes stood on the 9-m line opposing one attacker (another athlete). Each action was executed against another attacker, and the order of the attackers was random. The attacker tried to throw a goal after a double pass with the coach (within a marked zone of $3 \mathrm{~m}$ in width), while the participants tried to prevent the attackers from scoring and to gain possession of the ball. Each participant conducted each of the 3 movement tasks 3 times at each measurement. The order of the tasks was counterbalanced. All movements were videotaped. Stand throws were recorded from the side of the throwing arm; body feints and the defence against an attacker were recorded from the middle line of the handball field. These angles were chosen because they provide an optimal view for rating relevant performance indicators (e.g., stand throw: elbow on shoulder height; body feint: attacking the gap on the left and then moving to the right; defence position: inclined position, raised arms with bent elbows).

\section{Intervention}

After the pre-test, the participating athletes completed 4 training interventions within 2 weeks. While one group practised, the other group played small-sided games on the other part of the handball field. The stepby-step and analogy groups alternately started with either practice or play in each training intervention. Further, the order of the practised movement tasks was counterbalanced. During each session, each group practised for 40 minutes; thus, each movement was practised for 10-15 minutes. Within these 10-15 minutes, each athlete performed 6-8 repetitions with 2-3 instruction/feedback situations. After 2 weeks, the post-test was conducted. The athletes had no training practice in the 2 weeks following the post-test (owing to Christmas holidays). The retention-test was performed in the first training session after this 2-week break. The tests and the interventions were led by a 26-year-old handball coach with 8 years of training experience. The conduction of the tests and the interventions were controlled by an independent observer, taking field notes of the teaching behaviour of the coach. The observer discussed these field notes with the coach after every unit, making sure that the coach did not prefer one instruction strategy and tried to teach both groups with the same motivation and effort.

\section{Step-by-step instruction}

Movements were demonstrated and described by using step-by-step movement rules (Table 1). These rules were developed by the coach and one more experienced handball coach (A-licence of the German Handball Federation, > 15 years of experience in youth handball) prior to the study.

Questions and direct instructions were used (e.g., 'Did your left shoulder point in the direction of the goal?,' 'Make a bigger step to the right,' 'Attack the ball with your left hand first') to provide direct feedback to the athletes.

\section{Analogy instruction}

For every movement task, an analogy instruction was developed prior to the conduction of the study by the same 2 coaches as mentioned above.

For the stand throw, the athletes were instructed to imagine a tattoo on their left shoulder (for the lefthanded players, on their right shoulder) that should be visible to the goalkeeper during every throwing motion. This was assumed to prepare the athletes for the necessary rotation of hip and trunk for a successful throwing movement. To get athletes to raise their elbow, an analogy was derived out of a well-known kid dance [14]. All participants knew this dance because it had been used in several warm-ups beforehand. Within this dance, the kids raised their arms, with elbows bent, up to the shoulder height to show that they were 'strong like a tiger'. The analogy derived on this basis was the following: 'Show the goalkeeper your tattoo and show him with your arm that you are strong like a tiger'. 
F. Fasold, L. Houseman, B. Noël, S. Klatt, Instructions in handball

Table 1. Examples of the step-by-step instructions for the 3 movement tasks. All instructions were for right-handed athletes or against a right-handed attacker in the defence position. If the athletes were left-handed or defended against a left-hander, the instructions were adjusted

\begin{tabular}{|c|c|c|}
\hline Stand throw & Body feint & Defence position \\
\hline $\begin{array}{l}\text { 'After catching the ball, your left } \\
\text { shoulder should point in the direction } \\
\text { of the goal' } \\
\text { 'Move the ball behind your head' }\end{array}$ & $\begin{array}{l}\text { 'After catching the ball, make your } \\
\text { steps: left, right, left' } \\
\text { 'Make the first step to the open space } \\
\text { on the left side of the Air-Body' }\end{array}$ & $\begin{array}{l}\text { 'Move your left hand and foot, } \\
\text { pointing in direction of the throwing } \\
\text { arm of your opponent' } \\
\text { 'Attack the ball with your left hand' }\end{array}$ \\
\hline $\begin{array}{l}\text { 'Raise your right elbow to or over } \\
\text { shoulder height' }\end{array}$ & $\begin{array}{l}\text { 'Move by making a fast and big step } \\
\text { with your right foot' }\end{array}$ & $\begin{array}{l}\text { 'Attack the body with your right hand' } \\
\text { 'Keep your elbows bent' }\end{array}$ \\
\hline $\begin{array}{l}\text { 'Let your arm point in the direction } \\
\text { of the goal after ball release' } \\
\text {... }\end{array}$ & $\begin{array}{l}\text { 'Make the last step in the direction } \\
\text { of the goal' } \\
\text {... }\end{array}$ & .. \\
\hline
\end{tabular}

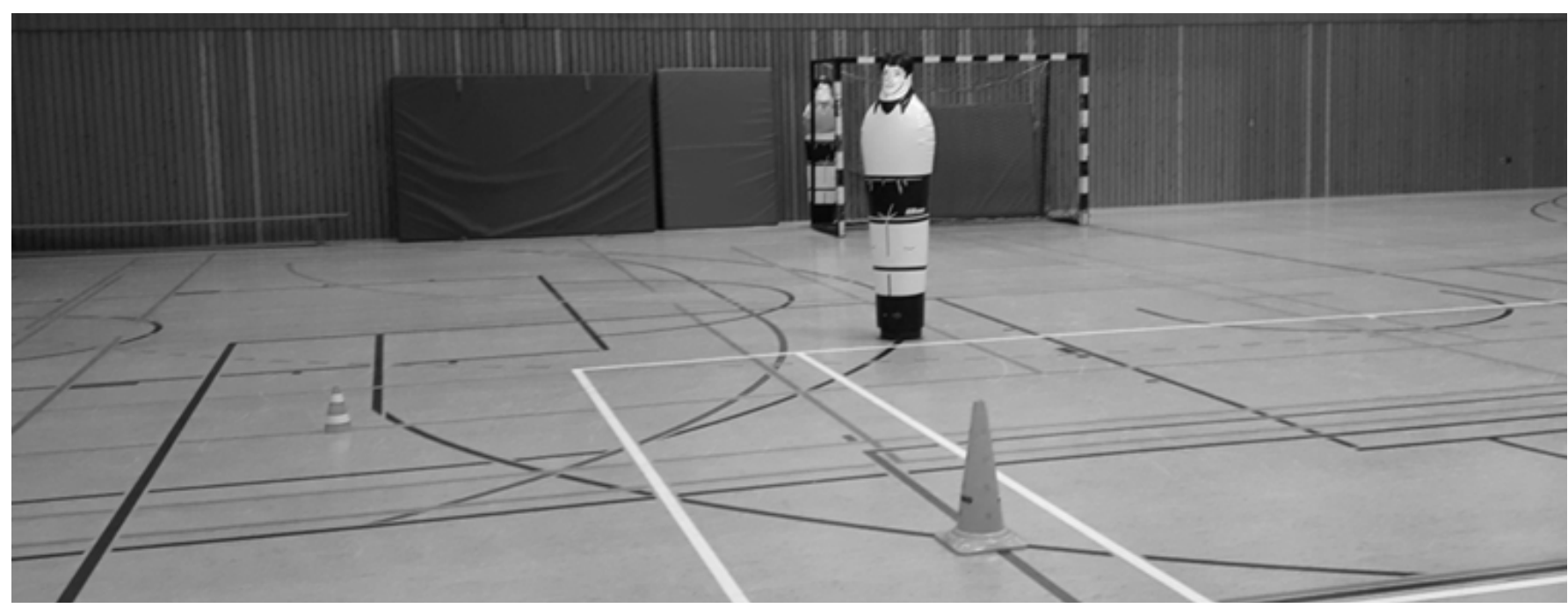

Figure 1. Photograph of the training equipment for the analogy 'Say "Hello” to Willi'

For the body feint analogy, an Air-Body wearing a training shirt was placed behind the goal (Figure 1). The participants called this Air-Body Willi. The derived analogy was: 'After catching the ball, say "Hello" to Willi and then quickly move away from Willi'. We expected that the athletes would first move towards the left space next to the Air-Body (defender) and then, with a fast change of direction, move to the right space and attack the goal.

For the defence position, the experimenter wrote the word 'Wait' on the palm of the left hand and the word 'Stop' on the palm of the right hand of each athlete, using a pen (Figure 2) (for the left-handed players, the sides of the words were changed). The derived instruction was that the attacker had to read out 'Wait, stop' and say 'The ball is mine'. This analogy was assumed to ensure raised arms and an inclined position
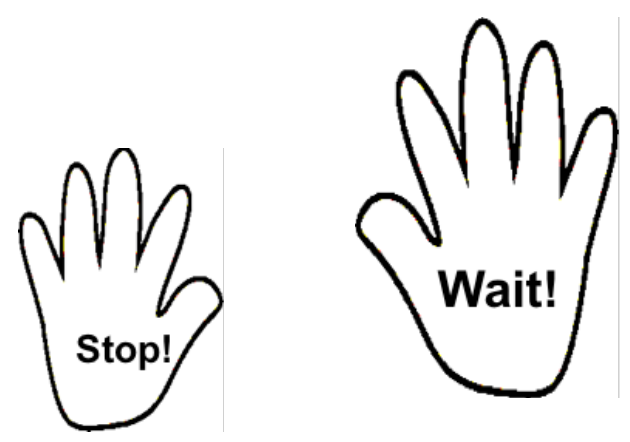

Figure 2. Preparation of the athletes' hands for the analogy 'Wait, stop, the ball is mine'

(left hand and body half attack the right-handed attacker first).

Questions and direct instructions based on these analogies were used to provide feedback ('Did the goalkeeper see your tattoo?,' 'How strong was the tiger?,' 'Willi is sad because you didn't say "Hello", 'Was "Wait" 
the first word the attacker could read?') within the intervention. These instructions were, like the step-bystep ones, developed prior to the study.

\section{Performance rating}

The video footage of the athletes' actions during every different task was shown to the raters (in sequences of 3 repetitions of an athlete). This resulted in 9 sequences (throw, feint, defence $\times$ pre-, post-, retention-test) per athlete and 240 sequences in total. We developed a computer program to present these sequences in 3 blocks (throw, feint, defence). The order of the presentation of the blocks was randomized. At the beginning of each block, the participating raters were instructed to rate the execution/performance of the athletes in the respective movement tasks. Furthermore, at the beginning of each block, the program presented one of the best and one of the worst performances (of all trials) to avoid calibration effects in the ratings [15]. While preparing the video sequences, the best and worst performances were identified by the coach. After calibration, the video sequences were presented in a random order. After each sequence, the raters judged the performance with the national university grading system $(1.0,1.3,1.7,2.0,2.3, \ldots, 5.0$; 1.0 represented the best, 5.0 the worst grade) by typing the grade in a box on the screen. After the grade confirmation by a key press, the program presented the next performance. After each of the 3 blocks, the raters could take a short break. On average, the rating of the video sequences took 96 minutes. The interrater reliability was satisfying across all task conditions, learning phases, and instruction conditions (all Cronbach's a values $>0.92$ ).

\section{Statistical analysis}

To analyse the effects of the factors (task, learning phase, instruction) on the dependent variable performance improvement, we mainly differentiated between improved and not improved performances. The pre-test performances were considered as baseline values and were compared with the post- and retention-test performances. Because 3 rating outcomes were possible for each comparison (improved, decreased, equal), we assumed that an overall rate of ca. $33.33 \%$ performance increase indicated a non-learning effect. In the following analysis, an equal or a decreased performance was considered as not improved. We conducted Barnard's unconditional test [16] for the frequency comparisons using $2 \times 2$ tables and calculated Cramer's $\varphi$ as effect size.

\section{Ethical approval}

The research related to human use has complied with all the relevant national regulations and institutional policies, has followed the tenets of the Declaration of Helsinki, and has been approved by the authors' institutional review board or an equivalent committee.

\section{Informed consent}

The athletes' parents were verbally informed about the procedure and the purpose of the study. Their written informed consent was obtained prior to the athletes' participation. After conducting the retention-test, all participating athletes and their parents were informed about the content of the study. The participating raters provided their written consent before the conduction of the study and were informed about the rationale after its conclusion.

\section{Results}

Owing to missed training sessions, missed tests, or dropouts, only the data of 25 of the participating athletes were used for analysis. Both groups were homogenous in height (step-by-step: $M=143.17 \mathrm{~cm}, S D=8.59$; analogy: $M=142.77 \mathrm{~cm}, S D=8.88 ; t(23)=0.11 ; p=$ 0.91 ) and weight (step-by-step: $M=33.33 \mathrm{~kg}, S D=5.56$; analogy: $M=34.69 \mathrm{~kg}, S D=4.13 ; t(23)=-0.69 ; p=$ 0.49 ). The athletes' gender distribution was similar across both groups (step-by-step: 4 females, 8 males; analogy: 4 females, 9 males).

\section{Base rate of learning}

To take into account the dependencies of the factors of task and learning phase, we calculated a base rate of learning from the pre-test to the retention-test; this base rate reflects the percentage of improved performances. Thus, the 1125 performance ratings of the pre-test performances were used as references for the retention-test ratings $(n=1125)$. The base rate of learning was $45.51 \%$ (improved performances ${ }^{1}$ ). All other frequency distributions in the experimental design were now compared with this base rate by using $2 \times 2$ tables (e.g., $n_{\text {impr. }}=512, n_{\text {not impr. }}=613, n_{\text {impr. }}=x$, $n_{\text {not impr. }}=x$; for a similar statistical approach, see Fasold et al. [15]). Furthermore, we controlled for the effects of the several factors by direct comparisons in $2 \times 2$ tables (e.g., throw $_{\text {impr. }}=n$, throw not impr. $=n$, feint $t_{\text {impr. }}$. $=n$, feint $t_{\text {not impr. }}=n$ ).

\footnotetext{
${ }^{1}$ The entire frequency distribution shows $45.51 \%$ improved, $32.80 \%$ decreased, and $21.96 \%$ equal performances in the base rate of learning.
} 
Task

We compared (independently of learning phase and instruction) the distribution of improved and not improved performances with the base rate for every task throw $_{\text {impr. }}=257$, throw $_{\text {not impr. }}=493$; feint $_{\text {impr. }}=$ 344, feint $_{\text {not impr. }}=406$; defence $_{\text {impr. }}=326$, defence $_{\text {not }}$ impr. $=424)$. We found significantly fewer improved performances, meaning a non-learning effect, in the throwing task (34.27\%, $p<0.001, \varphi=0.11$ ), whereas the learning effects in the other tasks equalled the base rate (feint: $45.87 \%, p=0.91, \varphi<0.00$; defence: $43.47 \%, p=0.50, \varphi=0.02)$.

\section{Learning phase}

Controlling for the factor of learning phase (independently of task and instruction), a non-learning effect was visible from the pre- to the post-test $(37.69 \%$, $n_{\text {impr. }}=424, n_{\text {not impr. }}=701, p<0.001, \varphi=0.07$ ), whereas the frequency distribution from the post- to the retention-test $\left(44.71 \%, n_{\text {impr. }}=503, n_{\text {not impr. }}=622\right.$, $p=0.70, \varphi=0.00$ ) was equal to the base rate (showing a learning effect from pre- to the retention-test).

\section{Instruction}

Testing the effect of the factor of instruction (independently of task and learning phase) against the base rate, we found fewer improved performances in the step-by-step condition $\left(40.00 \%, n_{\text {impr. }}=432, n_{\text {not }}\right.$ impr. $=648, p<0.01, \varphi=0.05)$, whereas the analogy condition $\left(42.31 \%, n_{\text {impr. }}=495, n_{\text {not impr. }}=675\right)$ was similar to the base rate $(p=0.12, \varphi=0.03$. However, a direct comparison of the frequency of improved performance ratings between the 2 conditions did not reveal a statistical difference $(p=0.34, \varphi=0.03)$.

\section{Interactions}

The analyses of the frequencies of improved performances with respect to the interaction of the experimental design factors are shown in Figure 3.

\section{Throw}

Considering the throwing task, the rate of improved performances (learning rate) was lower than the base rate in both learning phases (pre- to post-test: step-by$s t e p_{\text {impr. }}=62$, step-by-step $p_{\text {not impr. }}=118$, analog $y_{\text {impr. }}=61$, analog $y_{\text {not impr. }}=134$; post- to retention-test: step-bystep $_{\text {impr. }}=67$, step-by-step $_{\text {not impr. }}=113$, analog $y_{\text {impr. }}=67$, analog $y_{\text {not impr. }}=128$ ). Direct comparisons of the instructions in the throwing task showed no significant differences for the first ( $p=0.59, \varphi=0.03$ ) or the second learning phase ( $p=0.61, \varphi=0.02)$. Moreover, the direct comparisons of the 2 learning phases within the groups revealed no differences (step-by-step: $p=$ $0.62, \varphi=0.02$; analogy: $p=0.59, \varphi=0.03$ ).

\section{Feint}

For the learning of the feint task between pre- and post-test, the step-by-step instructions were more effective (but not different from the base rate) than the analogy instructions, which were worse than the base rate (pre- to post-test: $s t e p-b y$-step impr. $=74$, step$b y$-ste $p_{\text {not impr. }}=106$, analog $y_{\text {impr. }}=53$, analogy $y_{\text {not impr. }}=$ 142; direct comparison of the instructions: $p<0.001$, $\varphi=0.14)$. However, both types of instructions led to a massive increase of improved performances in the second learning phase (Figure 3; post- to retention-test: step-by-ste $p_{\text {impr. }}=101$, step-by-step $p_{\text {not impr. }}=79$, analo$g y_{\text {impr. }}=116$, analog $y_{\text {not impr. }}=79$; no differences appeared in the direct comparison: $p=0.60, \varphi=0.03$ ) When directly comparing both learning phases (pre- to post- vs. post- to retention-test) within the groups, it was shown that the visible effect was statistically significant in both instruction conditions (step-by-step: $p<$ 0.001, $\varphi=0.15$; analogy: $p<0.0001, \varphi=0.32$ ).

\section{Defence}

For learning the defensive position, the use of stepby-step instructions led to a less improved performance, whereas the use of analogies led to significantly more improved performances in the first learning phase (Figure 3; pre- to post-test: step-by-step $p_{\text {impr. }}=64$, stepby-step not impr. $=116$, analog $y_{\text {impr. }}=110$, analog $y_{\text {not impr. }}$ $=85$ ). The direct comparison between the 2 instructions in the first learning phase validated this effect $(p<0.0001, \varphi=0.20)$. For the second learning phase, the percentage of improved performances in the stepby-step condition was identical to the first phase ( $p=1$, $\varphi=0$; post- to retention-test: step-by-step $p_{\text {impr. }}=64$, step$b y$-step $p_{\text {not impr. }}=116$ ). The number of improved performances in the analogies condition decreased significantly from the first to the second learning phase (post- to retention-test: analog $y_{\text {impr. }}=88$, analog $y_{\text {not impr. }}=107$; $p=0.02, \varphi=0.11)$. It was therefore similar to the base rate (in the second phase) and still higher than that in the step-by-step condition (Figure 3), although we have to state that the latter difference did not reach the level of significance ( $p=0.06, \varphi=0.09$ ). 


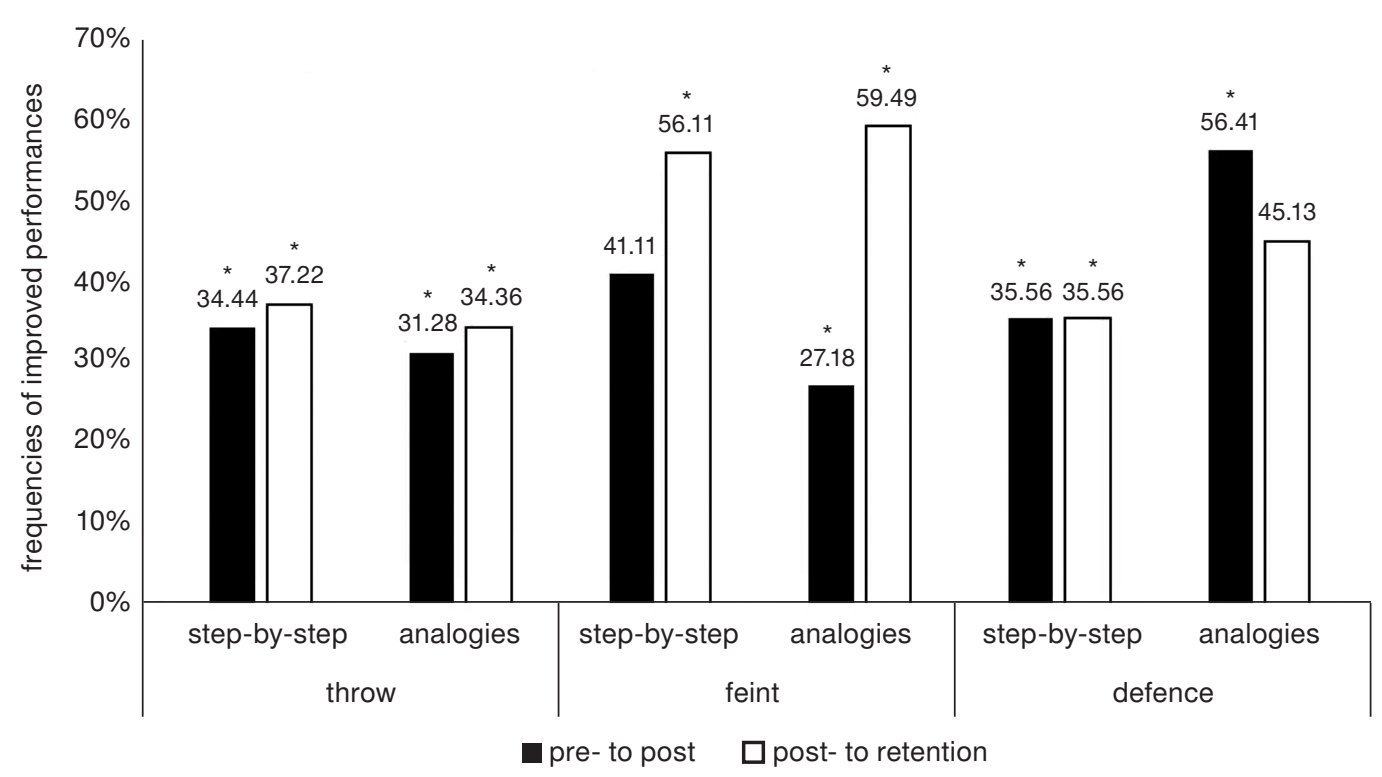

* significantly different from the base rate (45.51\%) with all $p<0.03$, all $\varphi>0.05$, without * all $p>0.35, \varphi<0.03$

Figure 3. Percentages of improved performances in both learning phases for each experimental factor

\section{Discussion}

The current study is the first to evaluate the use of explicit step-by-step and implicit analogical instructions among young athletes for specific skill acquisition in handball. As expected, both strategies had a similar positive effect on performance development, although the learning effects took time (second learning phase). Moreover, it was shown that handball skills could be trained in a pictorially metaphorical way. Although we were able to statistically verify the positive effect on learning/performance, we only found small effects $(\varphi \max =0.32,45.51 \%$ base rate of learning), and these effects were task-dependent. In the stand throw task, no differences and no learning effects could be observed. Because this non-effect was independent of instructions, we assume that the intervention phase was too short. In general, the stand throw is considered the most basic skill in handball, and all participating athletes were experienced in throwing a ball effectively. Thus, longer intervention periods seem to be necessary for improving the stand throw skill in skilled handball players. Compared with the stand throw, the body feint is a relatively new skill to be learned at this age and expertise level. Accordingly, both instruction strategies had a positive effect on performance development. Importantly, the stepby-step instructions were directly effective (pre- to post-test), whereas the analogy instructions took more time to be effective (decreased performance in the first and increased performance in the second learning phase relative to the base rate). Intriguingly, the perfor- mance in defending only improved with analogy instructions. The defence position was the only movement task with an active opponent. Thus, whereas the stand throw and body feint task conditions remained relatively stable, the defence position constituted a more complex task because of the active opponent trying to score a goal. As suggested in previous research, implicit learning strategies seem to be more effective in complex situations [11, 17]. One reason for this effect could be the lower requirements on working memory when the learning process is induced implicitly [8].

While the results of this study are of interest for coaching and training in handball, the current results are not completely new. That is, we replicated the results of several earlier investigations, pointing out that both learning strategies can be fruitful [4]. But the effectivity of a stimulated learning process is not the only crucial parameter for choosing a teaching method. Another important aspect is a teaching method efficiency, especially in teaching sports games like handball. If the game requires a lot of different skills and several athletes have to be coached simultaneously, time-saving feedback and instruction strategies can be necessary. Using metaphors or analogies could shorten the feedback processes and be helpful for developing an effective as well as an efficient coaching practice in sport and school. Our study could not validate the statement by Gabbett and Masters [18] that 'Analogies that truly exploit our visual abilities are probably more effective in coaching' (p. 571), but it did help to show that it may be worthwhile developing some simple analogies to foster skill acquisition in handball. 
The use of analogies in coaching does not necessarily lead to more effective learning; nevertheless, it potentially improves the efficiency of coaching practice.

Considering that we evaluated isolated movement tasks only, and not how learning effects transfer to actual in-game situations, further studies should focus on how different instruction types affect athletes' ingame performances. Furthermore, it would be interesting to analyse the effectivity/efficiency of mixed instructions (a combination of implicit and explicit instructions). A previous study focusing on the effects of mixed instructions on technical and tactical skills in basketball [19] indicated that this could be an effective coaching strategy as well. In particular, considering recent publications that criticize the conceptualization of implicit and explicit learning as isolated processes [6], mixing of instruction methods in sports practice should be investigated in more depth. Moreover, coaches' instructions and their general behaviour should not be evaluated out of context, that is, athletes' individual preferences and needs also have to be considered [20].

\section{Conclusions}

Summing up, we investigated the effects of step-bystep and of analogy instructions on young athletes' skill acquisition in handball for the first time. As a part of a short-term intervention, both coaching strategies improved the athletes' performances in a task in which they were relatively unexperienced (i.e., body feint). To conclude, for a more complex task (variable conditions: defending an active attacker), analogy instructions seem to be superior. This is in line with the results of previous research [17], and it is possibly the case because analogy instructions require less working memory processes. Even though no list of evaluated and functional analogies exists, the current study should motivate handball coaches to develop efficient and time-saving instructions. Furthermore, future research should focus on further variables/coaching strategies (e.g., mixed instructions), as well as the effectivity of analogy instructions in teaching group tactic behaviours (e.g., piston movement, pick and roll) to verify the findings of the present study and the existing research (for a review, see Wulf and Lewthwaite [6]) in more complex situations.

\section{Disclosure statement}

No author has any financial interest or received any financial benefit from this research.

\section{Conflict of interest}

The authors state no conflict of interest.

\section{References}

1. Karcher C, Buchheit M. On-court demands of elite handball, with special reference to playing positions. Sports Med. 2014;44(6):797-814; doi: 10.1007/s40279014-0164-z.

2. Wagner H, Finkenzeller T, Würth S, von Duvillard SP. Individual and team performance in team-handball: a review. J Sports Sci Med. 2014;13(4):808-816.

3. Raab M, Masters RSW, Maxwell J, Arnold A, Schlapkohl N, Poolton J. Discovery learning in sports: implicit or explicit processes? Int J Sport Exerc Psychol. 2009; 7(4):413-430; doi: 10.1080/1612197X.2009.9671917.

4. Tzetzis G, Lola AC. The effect of analogy, implicit, and explicit learning on anticipation in volleyball serving. Int J Sport Psychol. 2015;46(2):152-166; doi: 10.7352/ IJSP2015.46.152.

5. Hodges NJ, Franks IM. Modelling coaching practice: the role of instruction and demonstration. J Sports Sci. 2002;20(10):793-811; doi: 10.1080/02640410232067 5648.

6. Wulf G, Lewthwaite R. Optimizing performance through intrinsic motivation and attention for learning: the OPTIMAL theory of motor learning. Psychon Bull Rev. 2016;23(5):1382-1414; doi: 10.3758/s13423-0150999-9.

7. Sun R, Slusarz P, Terry C. The interaction of the explicit and the implicit in skill learning: a dual-process approach. Psychol Rev. 2005;112(1):159-192; doi: 10.1037/ 0033-295X.112.1.159.

8. Poolton JM, Zachry TL. So you want to learn implicitly? Coaching and learning through implicit motor learning techniques. Int J Sports Sci Coach. 2007;2(1):67-78; doi: 10.1260/174795407780367177.

9. Kal E, Prosée R, Winters M, van der Kamp J. Does implicit motor learning lead to greater automatization of motor skills compared to explicit motor learning? A systematic review. PLoS One. 2018;13(9):e0203591; doi: 10.1371/journal.pone.0203591.

10. Masters R. Skill learning the implicit way - say no more! In: Farrow D, Baker J, MacMahon C (eds.), Developing sporting expertise: researchers and coaches put theory into practice. Abingdon: Routledge; 2008; 89-103.

11. Liao CM, Masters RS. Analogy learning: a means to implicit motor learning. J Sports Sci. 2001;19(5):307-319; doi: 10.1080/02640410152006081.

12. Lam WK, Maxwell JP, Masters RS. Analogy versus explicit learning of a modified basketball shooting task: performance and kinematic outcomes. J Sports Sci. 2009;27(2):179-191;doi:10.1080/02640410802448764.

13. Schücker L, Hagemann N, Strauss B. Analogy vs. technical learning in a golf putting task: an analysis of performance outcomes and attentional processes under pressure. Hum Mov. 2013;14(2):175-184; doi: 10.2478/ humo-2013-0021. 
14. Such a beautiful day (an airman's song) [in German]. 2016. Available from: https://www.youtube.com/watch?v $=$ DDu5n9-ZkRE.

15. Fasold F, Memmert D, Unkelbach C. A theory-based intervention to prevent calibration effects in serial sport performance evaluations. Psychol Sport Exerc. 2015;18: 47-52; doi: 10.1016/j.psychsport.2015.01.001.

16. Mehrotra DV, Chan ISF, Berger RL. A cautionary note on exact unconditional inference for a difference between two independent binomial proportions. Biometrics. 2003;59(2):441-450; doi: 10.1111/1541-0420.00051.

17. Poolton JM, Maxwell JP, Masters RSW, Raab M. Benefits of an external focus of attention: common coding or conscious processing? J Sports Sci. 2006;24(1):89-99; doi: 10.1080/02640410500130854.

18. Gabbett T, Masters R. Challenges and solutions when applying implicit motor learning theory in a high performance sport environment: examples from rugby league. Int J Sports Sci Coach. 2011;6(4):567-575; doi: 10.1260/ 1747-9541.6.4.567.

19. Calábria Lopes M. Effectiveness of implicit and explicit learning processes: acquisition of tactical knowledge and motor skills in basketball [in German]. PhD thesis. Heidelberg: Heidelberg University; 2011.

20. Wałach-Biśta ZM. What do we want and what do we get from the coach? Preferred and perceived leadership in male and female team sports. Hum Mov. 2019;20(3): 38-47; doi: 10.5114/hm.2019.79734. 